Falkner, F. (1966) General considerations. Human Development, pp. 29-36. Ed. by F. Falkner. Saunders, Philadelphia. Falkner, F. (1978) Implications for growth in human twins. Human Growth, Volume I. Ed. by F. Falkner and J. M. Tanner. Plenum, New York (in press).

\section{Transthoracic expulsion of an} endobronchial foreign body

Sir,

A child expelled through the chest wall an endobronchial foreign body, an ear of grass (Hordeum murinum) which he had inhaled one week previously. I should like to know if such a case has been previously reported.*

P. BAgGio Division of Paediatrics, 'S. Lorenzo' Hospital, 36078 Valdagno, Italy.

*Two similar cases were reported from Greece by Choremis et al. in this journal $(1964,39,406)$; in both cases Hordeum murinum was also concerned.-Ed.

\title{
Referees 1977
}

We continue to depend heavily on the essential help we get from our referees who assess (and in many cases suggest significant improvements to) the papers submitted. This is the only opportunity the Editors have to acknowledge the work during the past year of the following.

$\begin{array}{lll}\text { Charlotte M. Anderson } & \text { P. S. Gardner } & \text { A. P. Norman } \\ \text { G. C. Arneil } & \text { F. Gianelli } & \text { J. M. Parkin } \\ \text { N. D. Barnes } & \text { S. Godfrey } & \text { A. D. Patrick } \\ \text { D. G. D. Barr } & \text { R. Goulding } & \text { P. Pinkerton } \\ \text { T. M. Barratt } & \text { D. B. Grant } & \text { P. E. Polani } \\ \text { J. D. Baum } & \text { W. Hamilton } & \text { M. A. Preece } \\ \text { P. F. Benson } & \text { J. T. Harries } & \text { D. N. Raine } \\ \text { J. F. R. Bentley } & \text { D. R. Harvey } & \text { H. A. Reid } \\ \text { C. Berry } & \text { R. G. Hendrickse } & \text { E. O. R. Reynolds } \\ \text { H. Bickel } & \text { R. Hoare } & \text { N. R. C. Roberton } \\ \text { Barbara Billings } & \text { K. S. Holt } & \text { A. J. Rook } \\ \text { B. D. Bower } & \text { D. Hull } & \text { H. M. Saxton } \\ \text { C. G. D. Brook } & \text { R. S. Illingworth } & \text { J. W. Scopes } \\ \text { M. J. Brueton } & \text { R. H. Jackson } & \text { J. E. S. Scott } \\ \text { D. N. Challacombe } & \text { R. S. Jones } & \text { Olive Scott } \\ \text { C. Chantler } & \text { M. C. Joseph } & \text { J. C. L. Shaw } \\ \text { Judith M. Chessells } & \text { E. G. Knox } & \text { E. A. Shinebourne } \\ \text { A. R. Chrispin } & \text { G. M. Komrower } & \text { Jean Smellie } \\ \text { C. A. Clarke } & \text { R. W. Smithells } \\ \text { J. J. Cogswell } & \text { J. A. Kuzemko } & \text { J. F. Soothill } \\ \text { K. W. Cross } & \text { P. Lachmann } & \text { J. B. P. Stephenson } \\ \text { Pamela A. Davies } & \text { B. M. Laurance } & \text { L. B. Strang } \\ \text { J. A. Davis } & \text { D. N. Lawson } & \text { J. M. Tanner } \\ \text { J. A. Dodge } & \text { J. Lorber } & \text { H. K. A. Visser } \\ \text { S. Doxiadis } & \text { D. MacCarthy } & \text { J. A. Walker-Smith } \\ \text { J. A. Dudgeon } & \text { J. A. Macfarlane } & \text { Sheila J. Wallace } \\ \text { P. M. Dunn } & \text { A. S. McNeish } & \text { R. J. West } \\ \text { J. L. Emery } & \text { D. J. Matthew } & \text { R. H. Whitaker } \\ \text { N. Evans } & \text { A. D. Milner } & \text { J. S. Wigglesworth } \\ \text { J. W. Farquhar } & \text { C. Bernadette Modell } & \text { J. Wilson } \\ \text { J. O. Forfar } & \text { Patricia Morris Jones } & \text { D. I. Williams } \\ \text { Louise Friedman } & \text { A. P. Mowat } & \text { M. L. N. Willoughby } \\ \text { M. Friedman } & \text { B. G. R. Neville } & \text { C. B. S. Wood } \\ \text { Gillian Gandy } & \text { H. H. Nixon } & \text { R. B. Zachary } \\ & & \\ & & \end{array}$

